\title{
A nomogram for predicting the risk of femoral pseudoaneurysm after neurointerventional procedures
}

\author{
Liangliang Yang ${ }^{1 \#}$, Puyuan Zhao ${ }^{1 \#}$, Bin Liu ${ }^{2 \#}$, Yang Gao ${ }^{1}$, Hao Zhou ${ }^{1}$, Qiuping Li $^{1}$, Yingchuan Jiang ${ }^{1}$, \\ Zhigang Yang ${ }^{1,3}$
}

${ }^{1}$ Department of Neurosurgery, Zhongshan Hospital Fudan University, Shanghai, China; ${ }^{2}$ Department of Neurology, Minhang Central Hospital, Shanghai, China; ${ }^{3}$ National Clinical Research Center for Interventional Medicine of China, Shanghai, China

Contributions: (I) Conception and design: L Yang, Z Yang; (II) Administrative support: Z Yang; (III) Provision of study materials or patients: L Yang, Z Yang; (IV) Collection and assembly of data: All authors; (V) Data analysis and interpretation: All authors; (VI) Manuscript writing: All authors; (VII) Final approval of manuscript: All authors.

\#These authors contributed equally to this work.

Correspondence to: Zhigang Yang. Department of Neurosurgery, Zhongshan Hospital Fudan University, 180 Fenglin Rd, Shanghai 200032, China. Email: yang.zhigang1@zs-hospital.sh.cn.

\begin{abstract}
Background: Because femoral pseudoaneurysm (FPA) is a dangerous complication of interventional procedures with a risk of life-threatening bleeding, our aim was to develop a predictive nomogram for FPA after neurointervention, and to suggest the best method for early identification of FPA.

Methods: We searched the PubMed database for literature addressing FPA after interventional procedures to analyze the risk factors, and we also reviewed the clinical data of patients from the Department of Neurosurgery who underwent neurointerventional procedures. Selected parameters were analyzed by univariate and multivariate logistic regression analysis. A nomogram was constructed using the independent risk factors by a multivariate regression model, and was validated by bootstrap resampling method, as well as receiver operating characteristic (ROC) curve, decision curve analysis (DCA) and calibration curve. The influence on the detection rate of FPA with Doppler ultrasound was also analyzed with Fisher's exact test.

Results: According to existing studies, female sex, diabetes and hypertension are major risk factors of FPA. Among 1,098 clinical patients, hypertension ( $\mathrm{P}=0.044)$, higher body mass index (BMI) $(\mathrm{P}=0.020)$, larger sheath size $(\mathrm{P}=0.049)$, puncture site hematoma $(\mathrm{P}=0.011)$ and closure failure $(\mathrm{P}=0.003)$ were identified as independent risk factors. The nomogram including these factors showed robust discrimination [C-index, 0.916; $95 \%$ confidence interval (CI): 0.810-1.022] with an area under the curve of 0.916. DCA indicated clinical utility, and the calibration curves showed an acceptable consistency. A significant improvement in the detection rate occurred when Doppler ultrasound was utilized $(\mathrm{P}=0.031)$.

Conclusions: The presented nomogram showed favorable predictive accuracy for FPA after neurointervention. We recommend ultrasound examination for patients at high risk of FPA evaluated by the nomogram.
\end{abstract}

Keywords: Femoral pseudoaneurysm (FPA); neurointervention; nomogram

Submitted Nov 12, 2021. Accepted for publication Dec 24, 2021.

doi: $10.21037 /$ atm-21-6508

View this article at: https://dx.doi.org/10.21037/atm-21-6508

\section{Introduction}

At present, most interventional operations are performed through femoral access, particularly when a larger sheath is required (1). Although radial access has been associated with favorable outcomes due to lower incidence of access-site complications, improved patient comfort and lower cost as compared with femoral access (2-4), there are still concerns about efficacy when there is higher procedural complexity 
and substantial guide catheter support is needed (5), which also increases the risk of arterial occlusion $(6,7)$. Iatrogenic pseudoaneurysm, compared with hematoma, arterial occlusion and arteriovenous fistula, is the most prevalent complication, with an incidence of $0.5-4.6 \%$ among cases of this access (8-10). However, femoral pseudoaneurysm (FPA) is a dangerous complication with a risk of lifethreatening bleeding. Pseudoaneurysm is a false aneurysm connected through a localized arterial wall perforation with a pseudocapsule confined and surrounded by adventitia and soft tissue, creating a cavity by the continuous blood flow (11). FPA is by far the most common iatrogenic pseudoaneurysm, with incidence to be $0.22-3.2 \%$.

Cerebrovascular disease is the top-ranked disease causing death in China $(12,13)$ and as a crucial treatment, neurointerventional procedures have developed rapidly, but compared with other interventional procedures, there is theoretically higher morbidity of FPA because of the extended access and procedure time. Most studies of FPA to date have considered cardiac intervention rather than neurointervention $(14,15)$, so research into the prevention and early diagnosis of this complication after neurointerventional procedures is particularly important. Nomograms to predict intracranial aneurysms have been reported before, while FPA risk factors remain unclear.

In this study, we retrospectively analyzed the data of patients who underwent a neurointervention between January 2017 and August 2020, combined with a literature review, to investigate the risk factors of FPA after neurointervention. We finally constructed a nomogram as an instrument to predict FPA morbidity, and summarize the methods for early diagnosis.

We present the following article in accordance with the TRIPOD reporting checklist (available at https://atm. amegroups.com/article/view/10.21037/atm-21-6508/rc).

\section{Methods}

\section{Literature review}

We performed an online search of the PubMed database from inception to June 2020 using the keywords "femoral", "pseudoaneurysm" and "intervention". Studies with complete data were included and case reports were excluded. Clinical and operating characteristics as well as risk factors in each study were collected. The search was limited to literature in the English language.

\section{Patient data}

All procedures performed in this study involving human participants were in accordance with the Declaration of Helsinki (as revised in 2013). The study was approved by institutional ethics board of Zhongshan Hospital Fudan University (No. B2021-137R). Individual consent for this retrospective analysis was waived.

From January 2017 to August 2020, we retrospectively reviewed 1,098 consecutive patients from the Department of Neurosurgery, Zhongshan Hospital, Fudan University, who underwent neurointerventional procedures. The retrieved information included sex and age, history of chronic diseases such as hypertension, diabetes, dyslipidemia and renal dysfunction, as well as platelet count, body mass index (BMI), history of smoking, and procedural details including sheath size, repeated puncture, puncture point hematoma, antiplatelet therapy, sheath exchange, and closure failure.

We defined a smoking history as $\geq 1$ cigarette/day for at least 6 months. Repeated puncture was defined as $\geq 1$ puncture within 1 month. Puncture point hematoma was defined as a conspicuous mass adjacent to the puncture site that was palpable. Closure failure was defined as persistent bleeding despite using a closure device. Antiplatelet therapy was administration of aspirin, clopidogrel, ticagrelor or cilostazol in the perioperative period. FPA was diagnosed by an experienced sonographer as a representative hypoechoic cyst beside the affected artery and colored blood flow from the catheterization site into the cyst on Doppler ultrasound.

\section{Statistical analysis}

The associations between the risk of FPA with the aforementioned clinical and procedural parameters were analyzed by univariate logistic regression analysis. These variables were then entered into a multivariate regression analysis to find the independent risks of FPA. Based on the multivariate logistic regression model, a nomogram to predict the risk of FPA was constructed.

Receiver operating characteristic (ROC) curve, decision curve analysis (DCA) and calibration curve were used to assess the performance of the nomogram. The area under the ROC curve (AUC) and Harrel's C-index were calculated to quantify the discriminative performance of the nomogram. Using the Hosmer-Lemeshow test, the predicted and actual probability of FPA were compared with a visual calibration plot to confirm the calibration of 
Table 1 Logistic multivariate regression analysis of femoral pseudoaneurysm risk

\begin{tabular}{|c|c|c|c|}
\hline \multirow{2}{*}{ Variables } & \multicolumn{3}{|c|}{ Multivariate analysis } \\
\hline & OR & $95 \% \mathrm{Cl}$ & $P$ value \\
\hline Hypertension & & & $0.044^{*}$ \\
\hline No & Reference & & \\
\hline Yes & 33.280 & $1.094-1,012.669$ & \\
\hline BMI & & & $0.020^{*}$ \\
\hline$\geq 27$ & 10.101 & $1.434-71.162$ & \\
\hline$<27$ & Reference & & \\
\hline Sheath size & & & $0.049^{*}$ \\
\hline$\geq 6 \mathrm{~F}$ & 30.601 & $1.016-922.066$ & \\
\hline$<6 \mathrm{~F}$ & Reference & & \\
\hline Hematoma & & & $0.011^{*}$ \\
\hline No & Reference & & \\
\hline Yes & 29.170 & $2.195-387.640$ & \\
\hline Closure failure & & & $0.003^{*}$ \\
\hline No & Reference & & \\
\hline Yes & 26.865 & $3.152-228.969$ & \\
\hline
\end{tabular}

${ }^{*}, \mathrm{P}<0.05$. OR, odds ratio; $\mathrm{Cl}$, confidence interval; $\mathrm{BMI}$, body mass index.

this nomogram. DCA was performed by quantifying the net benefits at different threshold probabilities to estimate the clinical utility of the nomogram. Moreover, as an internal validation, the nomogram was subjected to bootstrapping validation (1,000 bootstrap resamples) to assess its predictive accuracy.

Fisher's exact test analysis was performed for subgroups divided as Ultrasound and Non-ultrasound and their respective detection rates were calculated.

The statistical analyses and graphics were performed with IBM SPSS 20.0 (SPSS Inc., Armonk, NY, USA) and $\mathrm{R}$ software version 4.0.1 (The R Foundation for Statistical Computing, Vienna, Austria) with the rms package (for Nomogram construction and Calibration), pROC package (for ROC curve) and dca.R package (for DCA). $\mathrm{P}<0.05$ was considered to be statistically significant and the reported statistical significance levels were all two-sided.

\section{Results}

\section{Literature review}

A total of seven studies from the literature were included (14-20), all of them addressing cardiac intervention. The clinical and operating characteristics of all patients from these studies are shown in Table S1. The risk factors of FPA summarized from each study are shown in Table S2.

\section{Clinical characteristics and selected factors for model}

A total of 1,098 patients from our Department were included. Their clinical and operating characteristics were summarized and the univariate analysis demonstrated that high $\mathrm{BMI}(\mathrm{P}=0.046)$, large sheath size $(\mathrm{P}=0.033)$, puncture site hematoma $(\mathrm{P}=0.002)$ and closure failure $(\mathrm{P}<0.001)$ significantly correlated with the occurrence of FPA (Table S3).

\section{Development of the nomogram}

Multivariate logistic regression analysis identified hypertension $(\mathrm{P}=0.044)$, high $\mathrm{BMI}(\mathrm{P}=0.020)$, large sheath size $(\mathrm{P}=0.049)$, puncture site hematoma $(\mathrm{P}=0.011)$ and closure failure $(\mathrm{P}=0.003)$ as independent risk factors (Table 1). A model with the aforementioned risk factors predicting the risk of FPA was constructed and is presented as a nomogram (Figure 1). A score was calculated to assess the proportion of each variable. The total points were summed to predict the probability of FPA.

\section{Performance of the nomogram}

Using ROC analysis, our nomogram revealed robust discrimination with an AUC of 0.916 (Figure $2 A$ ). The calibration curve for the predictive ability of FPA showed a satisfying agreement between observation and prediction (Figure 2B). The C-index for the nomogram was 0.916 [95\% confidence interval (CI): 0.810-1.022], which was confirmed to be 0.874 with bootstrapping validation.

\section{Clinical application}

DCA of the nomogram revealed that if the threshold probability was $<33 \%$, the nomogram for predicting FPA 


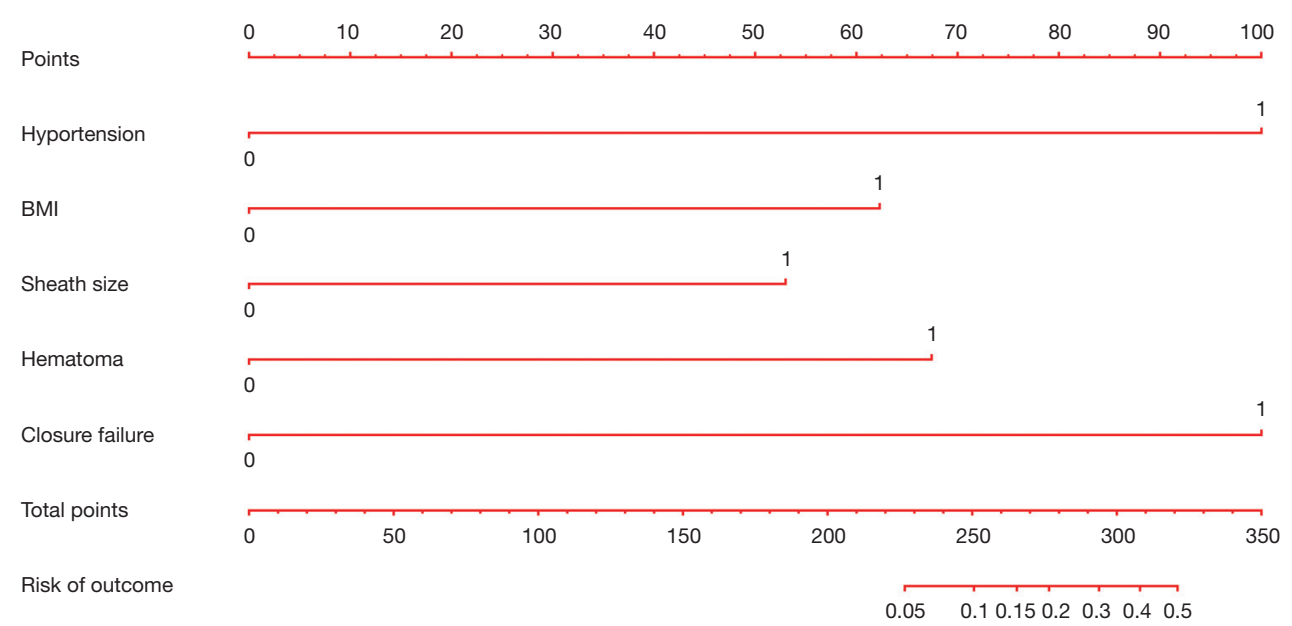

Figure 1 Nomogram for predicting the risk of femoral pseudoaneurysm (FPA) developed with the incorporation of hypertension, body mass index (BMI), sheath size, hematoma, and closure failure. Each score was calculated to assess the variable. The total points were summed to predict the probability of FPA. Harrell' concordance index $=0.916,95 \%$ confidence interval: 0.810-1.022.
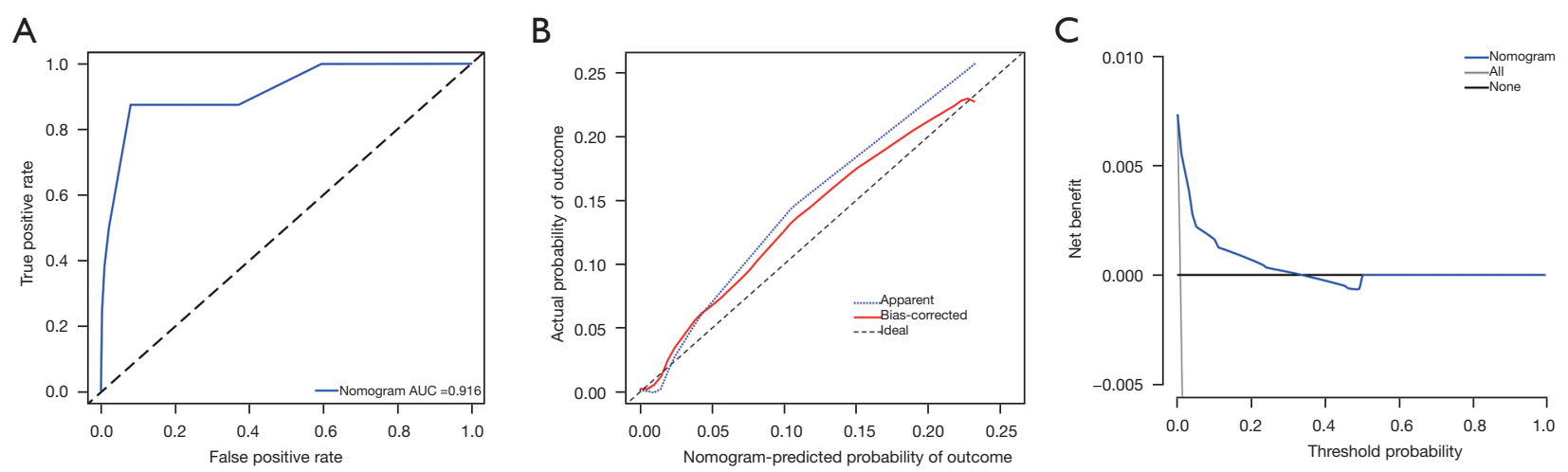

Figure 2 Validation performed by receiver operating characteristic (ROC) curve, decision curve analysis (DCA) and calibration curve. (A) ROC curve of the nomogram, area under the curve (AUC) $=0.916$, which showed a satisfying predictive efficiency. (B) Calibration curve showing consistency between the predicted probability (x-axis) with the observed real risk (y-axis) for femoral pseudoaneurysm (FPA). Perfect prediction is shown by the diagonal black dashed line. The blue dotted line shows the entire cohort. The red solid line represents bias corrected with bootstrapping. (C) DCA of the predictive instrument. The y-axis represents net benefit and the blue line is the nomogram. The gray is the supposition that all patients suffered from FPA while the black represents the supposition that none of the patients suffered from FPA.

added more benefit than treating either all or no patients (Figure 2C).

\section{Detection rate with Doppler ultrasound}

The detection rate of FPA for the non-ultrasound group was $0.4 \%$, and $1.6 \%$ for the Ultrasound group. Fisher's exact test showed a statistical significance for the group $(\mathrm{P}=0.031)$ (Table 2), which means Doppler ultrasound significantly improved the detection rate of FPA.

\section{Discussion}

FPA is a common access-site complication following interventional procedures. According to existing reports of cardiac intervention via femoral access, the incidence of iatrogenic pseudoaneurysm after diagnostic coronary angiography is $\sim 0.5 \%$, and as high as $4.6 \%$ in interventional 
Table 2 Fisher's exact test for the influence on the detection rate by Doppler ultrasound

\begin{tabular}{lccc}
\hline Ultrasound & Total & Positive $(n=8)$ & Negative $(n=1,090)$ \\
\hline No & 788 & $3(0.4 \%)$ & $785(99.6 \%)$ \\
Yes & 310 & $5(1.6 \%)$ & $305(98.4 \%)$ \\
\hline
\end{tabular}

*, $P<0.05$.

coronary procedures $(8,9)$. However, there are few reports on the incidence of FPA in neurointerventions. Only Ozono et al. reported an incidence of FPA after neurointervention with a large sheath (8-9 F) of $3.2 \%$ (4/126) (21). The complication rate of pseudoaneurysm is estimated to be $1.5 \%(1,008 / 69,266)$. According to our center's statistics, from January 2017 to August 2020, 634 cases of intracranial arteriography were performed and there were 464 cases of interventional treatment (for aneurysm, arteriovenous fistula, arteriovenous malformation, etc.). Among these, there were 8 cases of detectable pseudoaneurysms, with an incidence rate of $\sim 0.73 \%$.

Some studies reported the risk factors of FPA in cardiac intervention. Ates et al. reported hypertension, diabetes, high BMI, and a large sheath as major risk factors (14), Mlekusch et al. reported low platelet count (19), Badr et al. found diabetes along with non-utilization of avascular closure device were risk factors for FPA (18), and Erol et al. identified female sex, previous puncture, hypertension, anticoagulant therapy, and renal dysfunction as risk factors (17). Others suggested further examination if the patient has pain or a throbbing mass in the groin (15). This is truly important, but our contention is that the above symptoms are indicators rather than predictors of FPA, which is why we did not include them in our analysis. Based on our clinical practice, we suggest repeated puncture, hematoma formation at the puncture site, intraoperative exchange of long sheaths, and failure of vascular closure as feasible new predictable risk factors, and these were included in the univariate and multivariate analyses.

In the multivariate analysis, we found that hypertension, $\mathrm{BMI}>27$, sheath diameter $>6 \mathrm{~F}$, puncture site hematoma and closure failure were independent risk factors for FPA formation. According to clinical practice, these factors can be easily explained. Hypertension is an important factor affecting the elasticity and atherosclerosis of the blood vessel wall, and elevated femoral artery pressure will offset the compression force and cause continuous bleeding at the puncture point. Excessive fat (high BMI) and hematoma formation at the puncture point are considered to affect the accuracy and efficiency of puncture point compression. Larger sheath diameter is directly related to the size of the puncture breach. In addition, several researchers have reported a lower complication rate of the puncture site with the use of vascular closure devices compared with manual compression (22-24). Therefore, failure of closure increases the complications of the puncture site, including FPA. Based on clinical experience, exchanging a long sheath during the procedure would create repeated friction and injury of the localized arterial wall perforation. Repeated puncture reduces the healing of puncture points, and thus is considered an additional possible independent risk factor. However, along with low platelet count and antiplatelet therapy, repeated puncture and long sheath exchange did not show statistical significance in multivariate analysis, which probably resulted from the limited number of positive samples.

Nomograms are a tool to predict the risk and probability of an event. Due to the limitations of both data size and positive sample size, the nomogram model developed in this study was able to predict only $5-50 \%$ of FPA events, although $50 \%$ is a very dangerous probability in clinical practice. This suggests clinicians must closely monitor the puncture site and apply the correct management techniques, such as increasing the compression time and intensity. As for nomogram validation, the AUC of the ROC showed outstanding predictive performance, and the calibration curves results were also relatively acceptable. DCA showed that only when the probability was in the range of $0-33 \%$ could the nomogram provide a beneficial result. This also indicates that we should further expand the sample size or start a multicenter study to establish a more accurate and efficient nomogram prediction model, which is indeed our plan as the next step.

Our data analysis found that Doppler ultrasound of the femoral artery puncture point after neurointervention helped improve the detection rate of FPA (1.6\% vs. $0.4 \%)$, which suggests cases of missed FPA if ultrasound examination is not performed. Ultrasound is a diagnostic tool with great clinical significance due to its high sensitivity (94\%) and 
high specificity (97\%) (25). Despite being a cheap and straightforward technique, ultrasound may not be suitable for routine postoperative examination in certain centers, which may then benefit from using our nomogram. That is, for patients with higher risk predicted by the nomogram, ultrasound screening is undoubtedly required to reduce missed diagnosis of FPA, and facilitate early diagnosis and treatment. For asymptomatic "high-risk" patients with FPA, this model can greatly reduce the severe consequences of FPA progression caused by missed diagnosis.

At present, the treatment of FPA is still controversial. The mainstream techniques include ultrasound-guided compression (UGCT) (26), ultrasound-guided thrombin or collagen injection (27), interventional procedure with balloon, coil or stent and surgical resection also remains an option (28). Based on our FPA treatment experience and consideration of cost, we tend to firstly try UGCT and reserve surgical treatment for failed cases. In our series, 4 (50\%) of $8 \mathrm{FPA}$ patients failed to respond to compression and then underwent surgical resection. All patients had good outcomes.

To sum up, this predictive model has the potential to assist in clinical decision making on the necessity of a patient undergoing ultrasound examination, thus improving the detection rate and early treatment of FPA. It has been applied in our center on a small scale. We would increase the compression intensity and duration at the puncture site for those patients with FPA risk $>10 \%$. As our next step, we will further expand the sample size to establish a more accurate and efficient prediction model, and conduct a prospective multicenter study with further external validation to verify the accuracy of the model.

\section{Conclusions}

Our study identified a close relationship of FPA with hypertension, high BMI, large sheath size, puncture site hematoma and closure failure. The presented nomogram showed favorable predictive accuracy for FPA after neurointervention. We recommend Doppler ultrasound examination for those patients with high nomogram score to improve the detection rate of FPA and early treatment. Further verification of this instrument requires a larger sample size.

\section{Acknowledgments}

Funding: This study was supported by Foundation of
Shanghai Science and Technology Commission (No. 19ZR1450200).

\section{Footnote}

Reporting Checklist: The authors have completed the TRIPOD reporting checklist. Available at https://atm. amegroups.com/article/view/10.21037/atm-21-6508/rc

Data Sharing Statement: Available at https://atm.amegroups. com/article/view/10.21037/atm-21-6508/dss

Conflicts of Interest: All authors have completed the ICMJE uniform disclosure form (available at https://atm. amegroups.com/article/view/10.21037/atm-21-6508/coif). The authors have no conflicts of interest to declare.

Ethical Statement: The authors are accountable for all aspects of the work in ensuring that questions related to the accuracy or integrity of any part of the work are appropriately investigated and resolved. All procedures performed in this study involving human participants were in accordance with the Declaration of Helsinki (as revised in 2013). The study was approved by institutional ethics board of Zhongshan Hospital Fudan University (No. B2021$137 \mathrm{R})$. Individual consent for this retrospective analysis was waived.

Open Access Statement: This is an Open Access article distributed in accordance with the Creative Commons Attribution-NonCommercial-NoDerivs 4.0 International License (CC BY-NC-ND 4.0), which permits the noncommercial replication and distribution of the article with the strict proviso that no changes or edits are made and the original work is properly cited (including links to both the formal publication through the relevant DOI and the license). See: https://creativecommons.org/licenses/by-nc-nd/4.0/.

\section{References}

1. Ahmad F, Turner SA, Torrie P, et al. Iatrogenic femoral artery pseudoaneurysms--a review of current methods of diagnosis and treatment. Clin Radiol 2008;63:1310-6.

2. Jolly SS, Yusuf S, Cairns J, et al. Radial versus femoral access for coronary angiography and intervention in patients with acute coronary syndromes (RIVAL): a randomised, parallel group, multicentre trial. Lancet 2011;377:1409-20. 
3. Valgimigli M, Gagnor A, Calabró P, et al. Radial versus femoral access in patients with acute coronary syndromes undergoing invasive management: a randomised multicentre trial. Lancet 2015;385:2465-76.

4. Mason PJ, Shah B, Tamis-Holland JE, et al. An Update on Radial Artery Access and Best Practices for Transradial Coronary Angiography and Intervention in Acute Coronary Syndrome: A Scientific Statement From the American Heart Association. Circ Cardiovasc Interv 2018;11:e000035.

5. Megaly M, Karatasakis A, Abraham B, et al. Radial Versus Femoral Access in Chronic Total Occlusion Percutaneous Coronary Intervention. Circ Cardiovasc Interv 2019;12:e07778.

6. Joshi KC, Beer-Furlan A, Crowley RW, et al. Transradial approach for neurointerventions: a systematic review of the literature. J Neurointerv Surg 2020;12:886-92.

7. Majmundar N, Wilkinson DA, Catapano JS, et al. Reaccessing an occluded radial artery for neuroendovascular procedures: techniques and complication avoidance. J Neurointerv Surg 2021;13:942-5.

8. Schneider C, Malisius R, Küchler R, et al. A prospective study on ultrasound-guided percutaneous thrombin injection for treatment of iatrogenic post-catheterisation femoral pseudoaneurysms. Int J Cardiol 2009;131:356-61.

9. Li Y, Chen SH, Spiotta AM, et al. Lower complication rates associated with transradial versus transfemoral flow diverting stent placement. J Neurointerv Surg 2021;13:91-5.

10. Katzenschlager R, Ugurluoglu A, Ahmadi A, et al. Incidence of pseudoaneurysm after diagnostic and therapeutic angiography. Radiology 1995;195:463-6.

11. Baird RJ, Doran ML. The False Aneurysm. Can Med Assoc J 1964;91:281-4.

12. Xian W, Han B, Xia L, et al. Focusing on the premature death of redeployed miners in China: an analysis of causeof-death information from non-communicable diseases. Global Health 2019;15:7.

13. Wang YJ, Li ZX, Gu HQ, et al. China Stroke Statistics 2019: A Report From the National Center for Healthcare Quality Management in Neurological Diseases, China National Clinical Research Center for Neurological Diseases, the Chinese Stroke Association, National Center for Chronic and Non-communicable Disease Control and Prevention, Chinese Center for Disease Control and Prevention and Institute for Global Neuroscience and Stroke Collaborations. Stroke Vasc Neurol 2020;5:211-39. 14. Ates M, Sahin S, Konuralp C, et al. Evaluation of risk factors associated with femoral pseudoaneurysms after cardiac catheterization. J Vasc Surg 2006;43:520-4.

15. Popovic B, Freysz L, Chometon F, et al. Femoral pseudoaneurysms and current cardiac catheterization: evaluation of risk factors and treatment. Int J Cardiol 2010;141:75-80.

16. Ayhan E, Isik T, Uyarel H, et al. Femoral pseudoaneurysm in patients undergoing primary percutaneous coronary intervention for ST-elevation myocardial infarction: incidence, clinical course and risk factors. Int Angiol 2012;31:579-85.

17. Erol F, Arslan Ş, Yüksel İÖ, et al. Determinants of iatrogenic femoral pseudoaneurysm after cardiac catheterization or percutaneous coronary intervention via the femoral artery. Turk Kardiyol Dern Ars 2015;43:513-9.

18. Badr S, Kitabata H, Torguson R, et al. Incidence and correlates in the development of iatrogenic femoral pseudoaneurysm after percutaneous coronary interventions. J Interv Cardiol 2014;27:212-6.

19. Mlekusch W, Haumer M, Mlekusch I, et al. Prediction of iatrogenic pseudoaneurysm after percutaneous endovascular procedures. Radiology 2006;240:597-602.

20. Tamanaha Y, Sakakura K, Taniguchi Y, et al. Comparison of Postcatheterization Pseudoaneurysm between Brachial Access and Femoral Access. Int Heart J 2019;60:1030-6.

21. Ozono I, Sakamoto S, Okazaki T, et al. Management of post-puncture bleeding after neurointerventional procedures performed with a large-bore sheath introducer. J Clin Neurosci 2020;74:61-4.

22. Koreny M, Riedmüller E, Nikfardjam M, et al. Arterial puncture closing devices compared with standard manual compression after cardiac catheterization: systematic review and meta-analysis. JAMA 2004;291:350-7.

23. Geyik S, Yavuz K, Akgoz A, et al. The safety and efficacy of the Angio-Seal closure device in diagnostic and interventional neuroangiography setting: a singlecenter experience with 1,443 closures. Neuroradiology 2007;49:739-46.

24. Taha A, Walsh EK, Wright KA, et al. Safety and feasibility of a novel vascular closure device in neurointerventional procedures. Interv Neuroradiol 2013;19:353-8.

25. Coughlin BF, Paushter DM. Peripheral pseudoaneurysms: evaluation with duplex US. Radiology 1988;168:339-42.

26. Coley BD, Roberts AC, Fellmeth BD, et al.

Postangiographic femoral artery pseudoaneurysms: further experience with US-guided compression repair. Radiology 1995;194:307-11.

27. La Perna L, Olin JW, Goines D, et al. Ultrasound-guided 
thrombin injection for the treatment of postcatheterization pseudoaneurysms. Circulation 2000;102:2391-5.

28. Webber GW, Jang J, Gustavson S, et al. Contemporary management of postcatheterization pseudoaneurysms.

Cite this article as: Yang L, Zhao P, Liu B, Gao Y, Zhou H, Li Q, Jiang Y, Yang Z. A nomogram for predicting the risk of femoral pseudoaneurysm after neurointerventional procedures. Ann Transl Med 2022;10(2):62. doi: 10.21037/atm-21-6508
Circulation 2007;115:2666-74.

(English Language Editor: K. Brown) 


\section{Supplementary}

Table S1 Patients clinical and procedural characteristics from existing studies of FPA

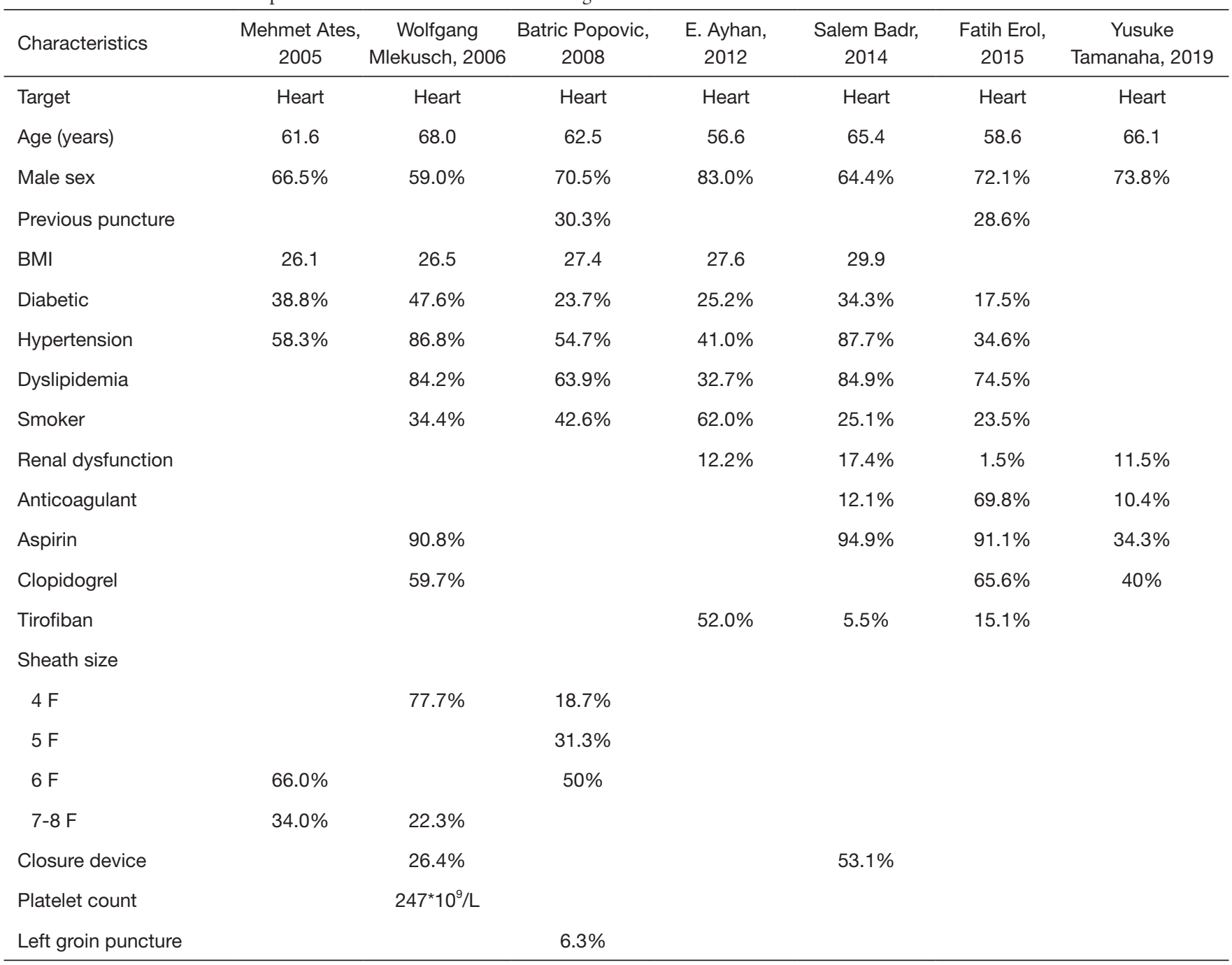

FPA, femoral pseudoaneurysm; BMI, body mass index. 
Table S2 Risk factors based on existing studies of FPA

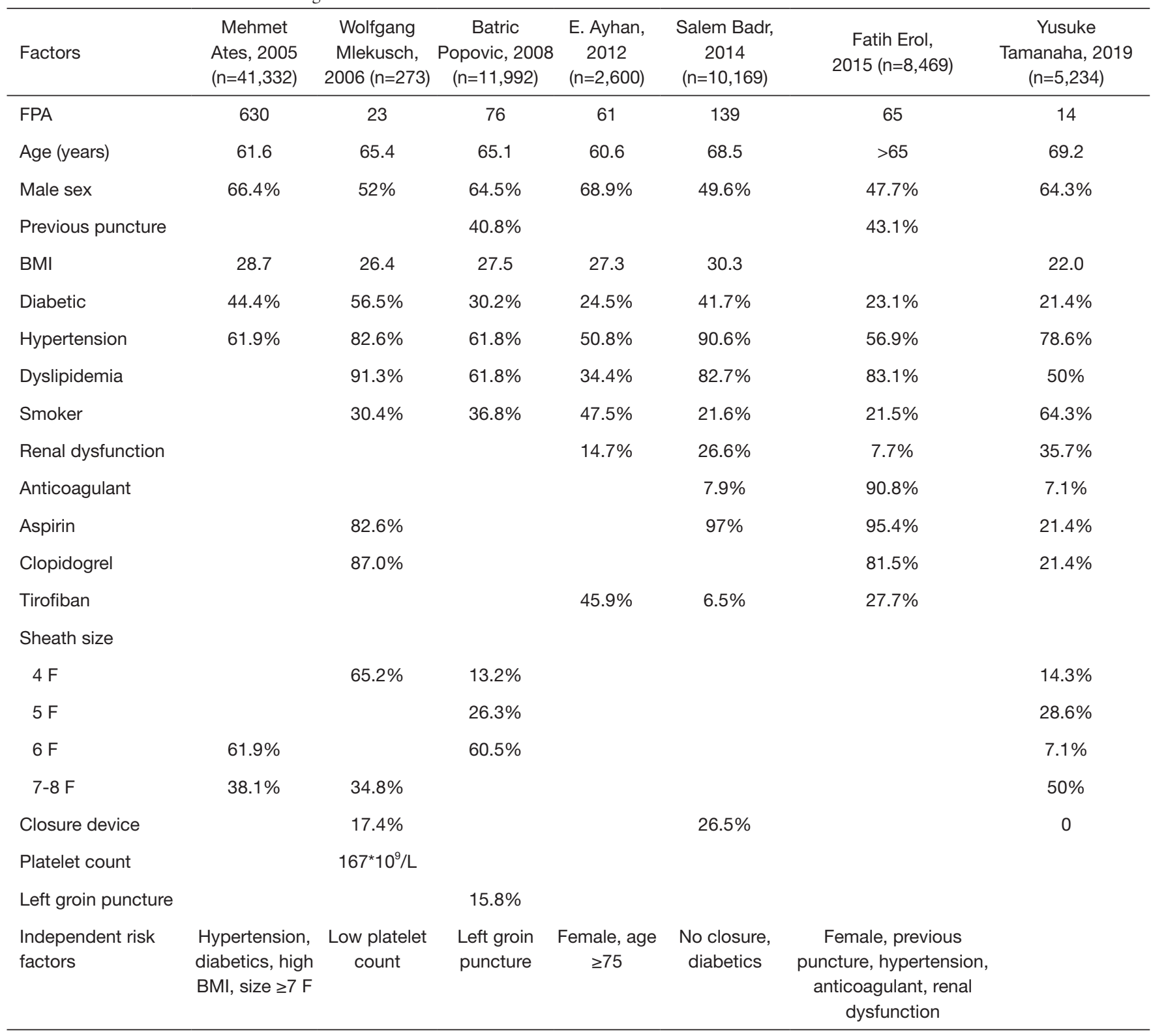

Numbers or proportions in table represent value from FPA group. FPA, femoral pseudoaneurysm; BMI, body mass index. 
Table S3 Patient clinical and procedural characteristics and univariable analysis of the risk of FPA in 1,098 patients after neurointervention

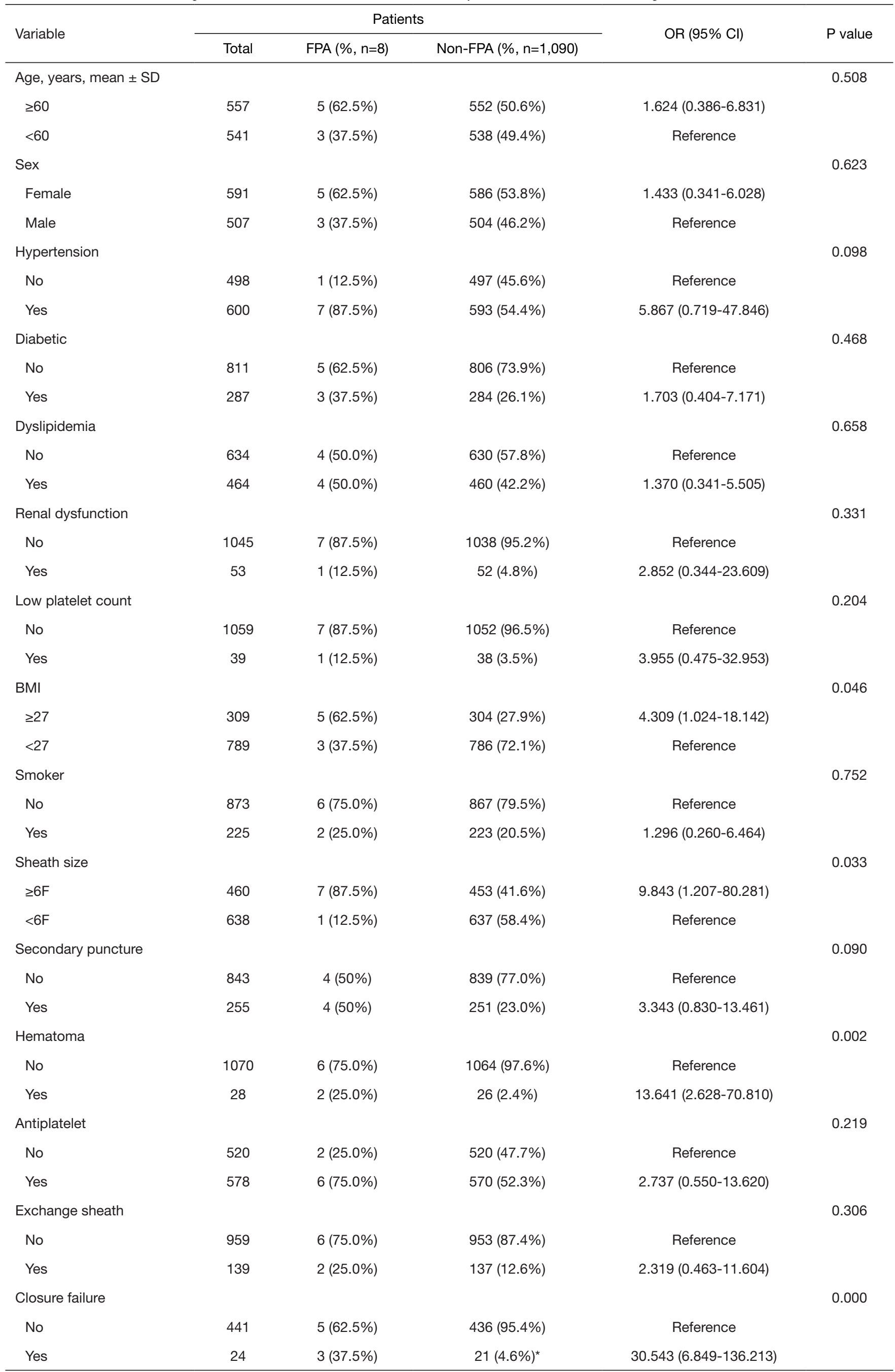

*, totally 457 in non-FPA group received closure, thus closure failure was 4.6\% (21/457). FPA, femoral pseudoaneurysm; OR, odds ratio; $\mathrm{Cl}$, confidence interval; SD, standard deviation; BMI, body mass index. 